\title{
An ID/Locator Separation Based Group Mobility Management in Wireless Body Area Network
}

\author{
Moneeb Gohar, ${ }^{1}$ Jin-Ghoo Choi, ${ }^{1}$ and Seok-Joo Koh ${ }^{2}$ \\ ${ }^{1}$ Department of Information and Communication Engineering, Yeungnam University, Gyeongsan 712-749, Republic of Korea \\ ${ }^{2}$ School of Computer Science and Engineering, Kyungpook National University, Daegu 702-701, Republic of Korea \\ Correspondence should be addressed to Jin-Ghoo Choi; jchoi@yu.ac.kr
}

Received 14 November 2014; Accepted 20 February 2015

Academic Editor: Bo-Wei Chen

Copyright (C) 2015 Moneeb Gohar et al. This is an open access article distributed under the Creative Commons Attribution License, which permits unrestricted use, distribution, and reproduction in any medium, provided the original work is properly cited.

\begin{abstract}
Mobility management in wireless sensor network is the most important factor to be considered for applications such as healthcare system. Recently, Identifier (ID)/Locator (LOC) separation based mobility management scheme has been proposed for wireless sensor network. However, it does not perform well in group-based mobility management in wireless body area network, and thus it tends to induce large registration, packet delivery, and handover delays. To overcome these limitations, we propose a group-based mobility management scheme based on ID/LOC separation concept for ID-based communications with location-based routing to reduce the number of control messages. In the proposed scheme, each sensor device has a globally unique device identifier (GDID) which contains the information of its home network domain. For handover support, each access gateway maintains its home GDID register (HGR) and visiting GDID register (VGR) which are used to keep the GDID-locator (LOC) mappings for primary mobile devices in the distributed manner. Besides, in the proposed scheme, only the coordinator will send Router Solicitation and Router Advertisement messages to reduce the control messages further. By numerical analysis, we show that the proposed scheme can significantly reduce the registration, packet delivery, and handover delays, compared to the existing schemes.
\end{abstract}

\section{Introduction}

Wireless body area networks (WBANs) are emerging as an important part of the daily life for ambient assistive living. A WBAN typically consists of lightweight, low power sensors that operate in the proximity of the human body. These sensors can be attached to human body or clothes [13 ] and can be used to measure the parameters associated with human body, typically observing physiological signals emanating from different body organs, body motions, and the surrounding environment. The measured values can be gathered and transmitted to the main server by using the IPv6 over low power wireless personal area network (6LoWPAN) $[4,5]$, in which it is possible to connect wireless sensor nodes to IPv6 networks. In mobile environments, it is required to provide these sensor nodes with mobility management, such as handover control.

Many IPv6-based mobility management protocols have attracted much interest in 6LoWPAN networks. We can classify the protocols into the host-based schemes and the network-based schemes. For example, Mobile IPv6 (MIPv6) is a host-based protocol [6] and Proxy Mobile IPv6 (PMIPv6) is a network-based protocol [7]. In hostbased mobility schemes, the sensor exchanges Binding Update $(B U)$ and Binding Acknowledgment (BA) messages with its Home Agent (HA), when it moves from one mobility domain to another. On the other hand, in network-based mobility schemes, when a sensor changes the domain, the protocols do not require any mobility-related signaling. Instead, a mobile access gateway (MAG) is responsible for detecting movement and exchanging signaling messages on behalf of the sensors. It is noted that PMIPv6 can be considered as the most suitable mobility scheme in 6LoWPAN-WBAN. However, the conventional PMIPv6 [7] scheme has a drawback that a lot of Proxy Binding Update (PBU) and Proxy Binding Ack (PBA) messages should be exchanged between LMA and MAGs for all body sensors. To enhance this conventional PMIP scheme, the PMIP-Group [8] was proposed, in which a single DeRegistration (DeReg) message is exchanged between MAG and LMA by aggregating the associated messages from all 
sensors. The PMIP-Coordinator scheme [9] was proposed for further enhancement of group-based mobility support in 6LoWPAN-based WBAN network. In this scheme, the Coordinator will communicate with MAG on behalf of the body sensor. The PMIP-Coordinator still has large registration and handover delay.

The Identifier (ID)/Locator (LOC) based mobility management scheme has been proposed for 6LoWPAN wireless sensor network [10]. In this scheme each primary mobile device (PMD) or 6LoWPAN sensor has a 128-bit global unique device identifier (GDID), which is used for end-toend communication, and a link-layer address can be used as the access identifier (AID). Each local network domain will have a local home mobility agent and a local visited mobility agent, which are configured based on the logical overlay network that supports the distributed mapping management. However, this scheme does not perform well in group-based wireless body area network, because each sensor sends Router Solicitation (RS) and Router Advertisement (RA) messages to PMD. In addition, for location update and discovery, the additional control messages are exchanged between access gateway and distributed local mapping agents. This tends to induce large registration and handover delays. For this reason, how to decrease the times of exchanging the control messages in case that a number of sensors are attached on one PMD is an important issue.

To overcome these limitations, we propose a groupbased mobility management scheme based on the ID/LOC separation concept. In the proposed scheme, each sensor and each primary mobile device has a globally unique device identifier (GDID) which contains the information of home network domain. For handover support, each access gateway maintains "home GDID register" and "visiting GDID register" that are used to keep the GDID-global locator (GLOC) mappings for primary mobile devices in the distributed manner. The proposed scheme also reduces the number of control messages, including $R S / R A$. This is because only the Coordinator will exchange $R S / R A$ messages with PMD, instead of each sensor.

The rest of this paper is organized as follows. In Section 2, we review the existing ID/LOC schemes for 6LoWPANWBAN networks. In Section 3, we describe the proposed ID/LOC scheme in detail. The performances of the existing and proposed schemes are analyzed in Section 4 in terms of the registration delay, packet delivery delay, and the handover delay. We provide the numerical results and discuss them in Section 5. Finally, we conclude this research in Section 6.

\section{Related Works}

In conventional PMIPv6 scheme [7], a lot of Proxy Binding Update (PBU) and Proxy Binding Ack (PBA) messages are exchanged between LMA and MAGs for all body sensors. To enhance this conventional PMIP scheme, the PMIP-Group [8] was proposed, in which a single De-Registration (DeReg) message is exchanged between MAG and LMA for all body sensors.
The PMIP-Coordinator scheme [9] was proposed for further enhancement of PMIP-Group scheme. In this scheme, the Coordinator will communicate with MAG on behalf of the body sensor.

As shown in Figure 1, when the Coordinator is detached from previous MAG (p-MAG), a single DeReg message is exchanged between p-MAG and LMA by aggregating the associated messages from all sensors.

When the Coordinator is attached to n-MAG, then it sends a single Router Solicitation (RS) message, which contains the associated group information, MN-IDs, and link-layer address, to n-MAG by way of PMD at a time. Upon reception of $R S$ messages from body sensors, nMAG will send the Authentication-Authorization-Accounting (AAA) query messages for authentication for all body sensors. After authentication, AAA server responds with $A A A$ reply messages, containing the LMA address, to n-MAG. Then, nMAG will send aggregated Proxy Binding Update (PBU) message to LMA for all body sensors. Now, LMA will perform the AAA query operation with AAA server by exchanging $A A A$ query and reply messages for each sensor. After that, LMA sends aggregated Proxy Binding ACK (PBA) message to $\mathrm{n}$ $M A G$ in response to the respective aggregated $P B U$ message. Then, n-MAG responds with a Router Advertisement (RA) message to the Coordinator in response to the $R S$ message.

If PMD wants to communicate with the corresponding PMD (C-PMD), then PMD will send a data packet to LMA directly and LMA will forward the data packet to corresponding MAG (c-MAG) and further to C-PMD.

The ID/LOC-based mobility management scheme has been proposed for 6LoWPAN wireless sensor network [10]. In this paper, we will consider it for 6LoWPAN-based wireless body area network. In the ID/LOC scheme, each primary mobile device (PMD), such as smartphone or tablet PC and 6LoWPAN sensor, has a 128-bit globally unique device identifier (GDID), which is used for end-to-end communication, and a link-layer addresses can be used as the access identifier (AID). Each local network domain will have the home and visited distributed local mobility management agents, which are based on the logical overlay network that supports the distributed mapping management. The device ID can be generated through cryptographical generated address (CGA).

As shown in Figure 2, when the body sensors are attached to PMD, then all body sensors generate their IDs and send Router Solicitation (RS) messages to PMD. Upon reception of $R S$ messages from body sensors, PMD will send the Location Update Request to access gateway (AGW). Then, the AGW will update its GDID-global locator (GLOC) mapping table and also GDID-access identifier (AID) mapping table. After that, the AGW responds with Location Update Response message to PMD. After location update, the AGW also performs the Location Update Request and Response messages with distributed local mapping agent (DLMA) for adding GDID-GLOC mapping for global communication.

When PMD moves from the previous access gateway ( $\mathrm{p}$ AGW) to a new access gateway (n-AGW), the PMD will send Attachment Trigger to n-AGW. After Attachment Trigger, n-AGW sends Device Context Request message to p-AGW. 


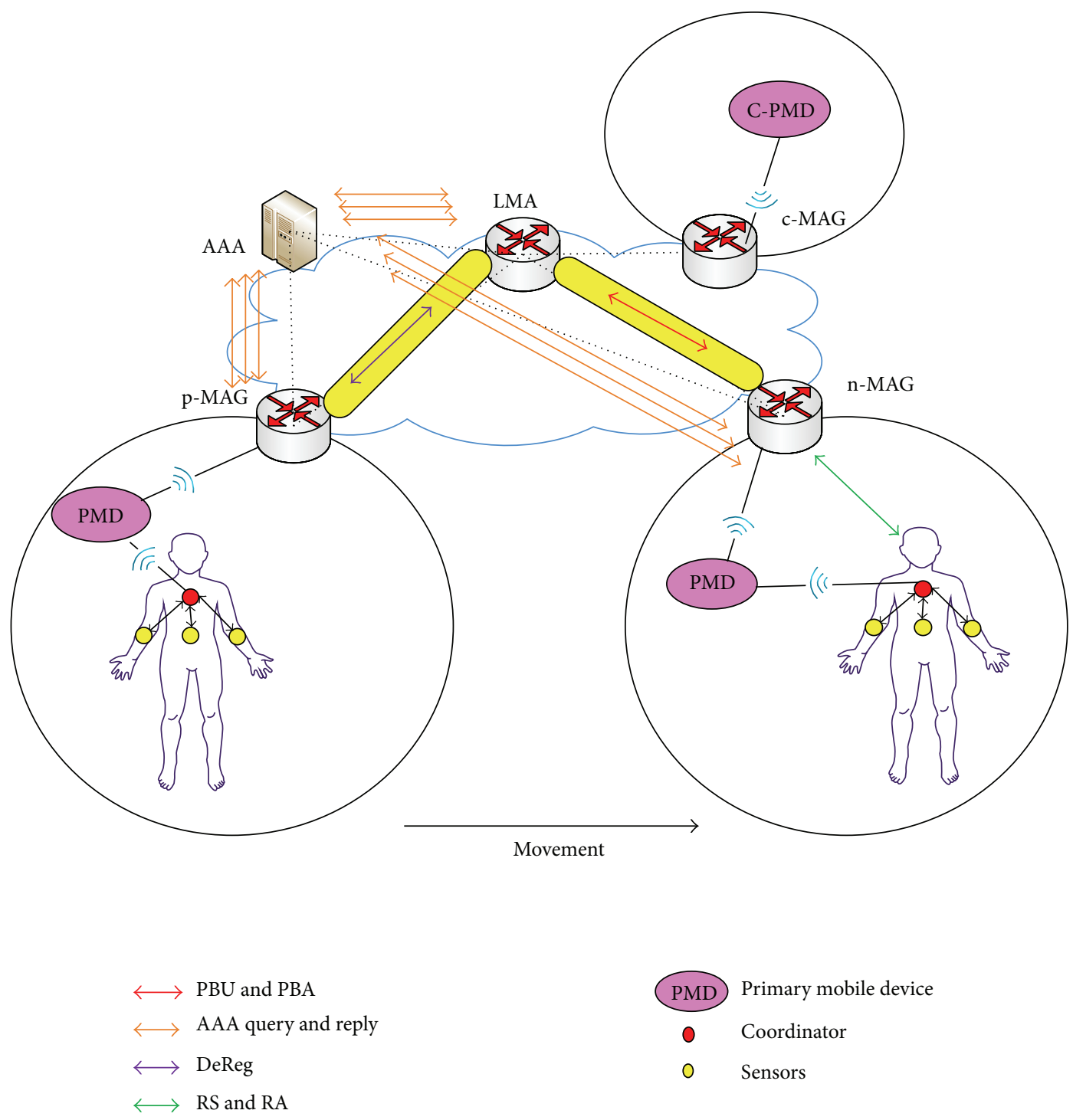

FIgURE 1: PMIP-Coordinator.

Then, p-AGW will send the Location Update Request message to home DLMA (H-DLMA). The H-DLMA updates the information and responds with Location Update Response message to p-AGW. After receiving the Location Update Response, the p-AGW will send Device Context Reply message to $\mathrm{n}$-AGW.

As shown in Figure 3, PMD wants to communicate with a particular PMD that is residing in the corresponding gateway (c-AGW). The PMD will send a Device ID Request message to AGW. After receiving the Device ID Request from PMD, the AGW will look up its mapping table, whether the request ID exists or not. If there is no information, then AGW sends a Location Discovery Request message to corresponding DLMA (C-DLMA). The C-DLMA will look up its mapping table and reply with a Location Discovery Response message to AGW. Upon the receipt of the Location Discovery Response message from the CDLMA, the AGW will add the information in its mapping table. Then, the AGW sends Route Setup Request message to c-AGW. After that, the c-AGW performs the GDID Discovery Query and Reply messages with corresponding PMD (C-PMD). After that, the c-AGW responds with Route Setup Complete message to AGW. After that, the AGW responds with Device ID Response message to PMD. Now, the data packets will be forwarded to C-PMD via AGW and c-AGW.

\section{Proposed Scheme}

In this section, we describe the proposed ID/LOC-based 6LoWPAN-WBAN mobility management scheme. To this end, first we need to specify the network model.

3.1. Network Model. The proposed ID/LOC-based 6LoWPAN wireless body area network mobility management scheme is shown in Figure 4. In the model, we consider 
TABLE 1: Comparison of mobility management architectures.

\begin{tabular}{lccc}
\hline Relevant protocols & PMIP-Coordinator [9] & ID-LOC-based scheme [10] & Proposed scheme \\
\hline Mobility agent & LMA & DLMA, AGW & AGW \\
Identifier/Locators & HoA, CoA & GDID, AID, GLOC & GDID, GLOC, LLOC (AID) \\
RS/RA message exchange & $\begin{array}{c}\text { Between coordinator and PMD } \\
\text { (one time) }\end{array}$ & $\begin{array}{c}\text { Between body sensors and PMD } \\
\text { (each body sensor) }\end{array}$ & $\begin{array}{c}\text { Between Coordinator and PMD } \\
\text { (one time) }\end{array}$ \\
\hline
\end{tabular}

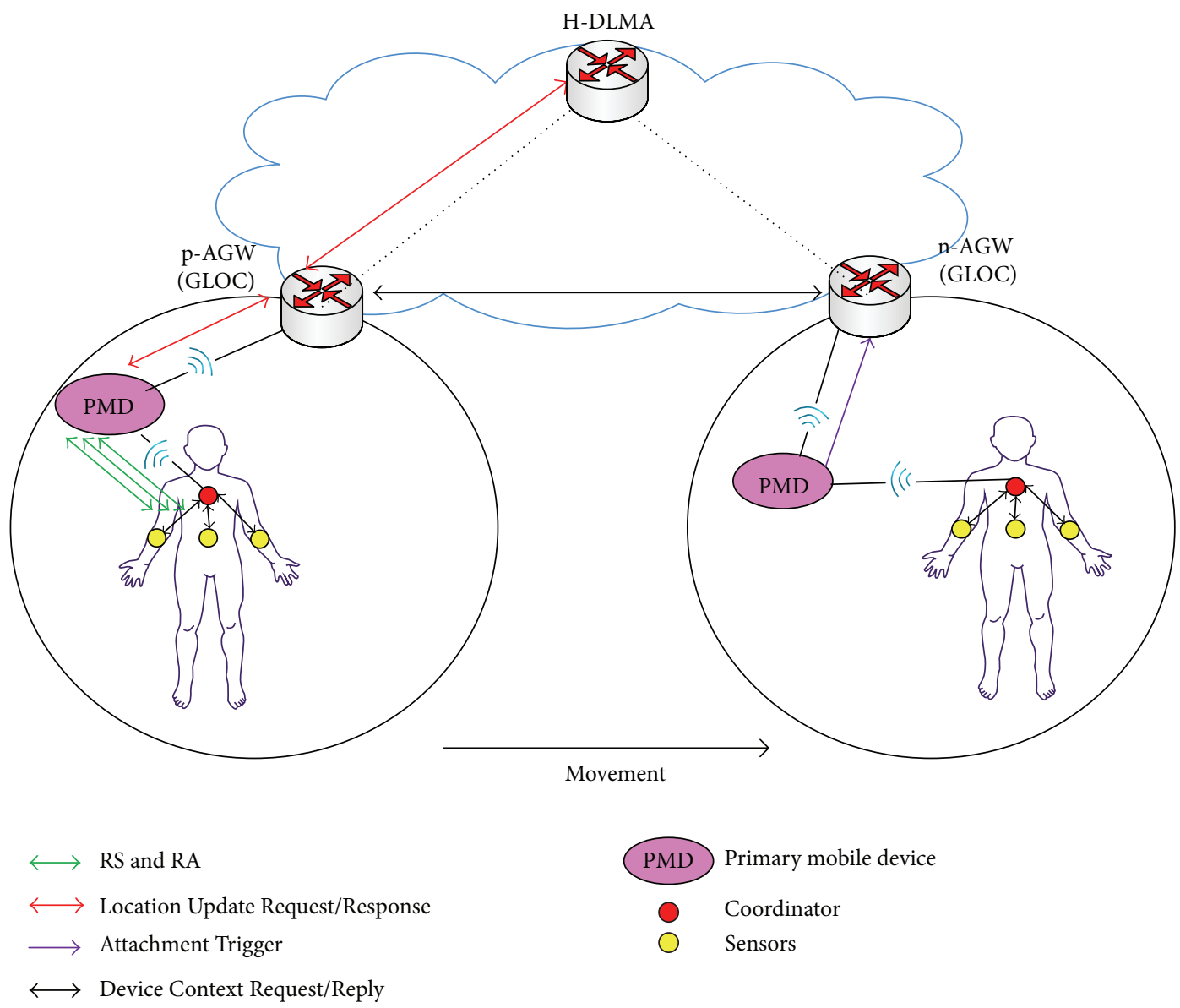

FIGURE 2: ID-LOC registration and handover operations.

a group of 6LoWPAN sensors that are attached to human body, and one of them acts as Coordinator and only the Coordinator can exchange the control signaling messages with the primary mobile device (PMD). In the proposed scheme, each sensor or PMD has a 128-bit globally unique device identifier (GDID) [10]. The link-layer addresses can be used as the access identifier (AID). The GDID contains the information about its home network domain. As for locators, the location of PMDs is identified by local locators (LLOC) and global locators (GLOC). The local locators are the AIDs of PMDs, and it is used within the home domain. The GLOC represents the IP address of access gateways (AGW), and it is used for interdomain communication. Each AGW keeps home GDID register (HGR) and visiting GDID register (VGR). HGR keeps track of the GDID-LOC mapping information for PMDs and VGR maintain the list of GDIDLLOC mapping information for the visited PMDs.
In the proposed scheme, only one time Router Solicitation (RS) and Router Advertisement (RA) messages are sent by Coordinator and thus reduce lots of control messages.

Initially, the PMD communicates with correspondent PMD (C-PMD) in the previous AGW (p-AGW) domain. Now, the PMD moves to a new AGW (n-AGW) by handover. In addition, we assume that each PMD moves around only within its home domain.

3.2. Comparison of Existing and Proposed Schemes. Before describing the proposed scheme in detail, we compare the considered mobility management schemes in the architectural perspective in Table 1.

In the viewpoint of the mobility management, PMIPCoordinator is the centralized architectures, in which all the control and data traffic are processed by a centralized agent such as LMA. Data packets are delivered to the centralized 


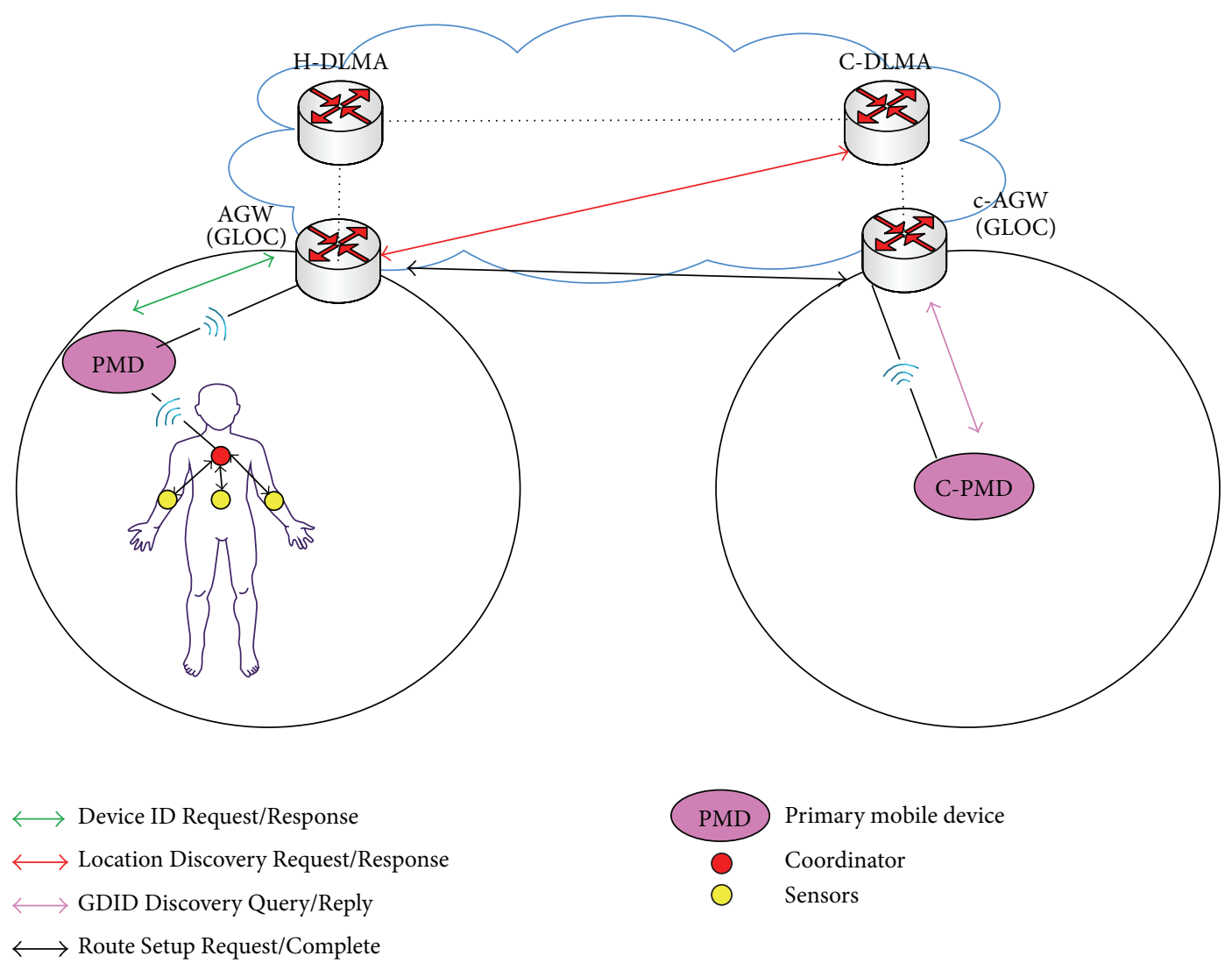

FIGURE 3: ID-LOC packet delivery operations.

agents first and forwarded to the corresponding host. In PMIP-Coordinator, the identifier corresponds to HoA and the locator does CoA. In PMIP-Coordinator, the Coordinator will exchange $R S / R A$ messages only one time with mobile access gateway (MAG) on behalf of the body sensors.

In ID-LOC-based scheme, the GDID is used as identifier, and AID and GLOC are used for locators. The DLMA and AGW are used to manage the mobility of PMDs. In ID-LOCbased scheme, the body sensors exchange RS/RA messages with PMD.

On the other hand, in the proposed schemes, the GDID is used as identifier and LLOC and GLOC are used for locators. The AGW manages the mobility for PMDs. In the proposed scheme, the Coordinator exchanges $R S / R A$ messages with PMD only one time on behalf of body sensors. The proposed scheme is described in the subsequent sections.

3.3. Initial Registration. The initial registration procedure of the proposed scheme is shown in Figure 5.

In the figure, when the Coordinator is attached to PMD, the Coordinator sends a Router Solicitation (RS) message containing the information on the group, MN-IDs (GDID), and Link-Layer Addresses (AIDs) to PMD (Step 1). Upon reception of the $R S$ message from the Coordinator, the PMD responds with $R A$ message to Coordinator (Step 2). Then, PMD sends Location Update Request message to AGW. On the reception of this message, the AGW will update its home GDID register (HGR) which maintains GDID-LOC mapping
TABLE 2: Home GDID register (HGR).

\begin{tabular}{lccc}
\hline Number & ID & LOC & Domain \\
\hline 1 & GDID1 & LLOC (AID) of PMD, & Home \\
2 & GDID2 & GLOC of AGW & Visiting \\
3 & $\ldots$ & $\ldots$ & $\ldots$ \\
\hline
\end{tabular}

table as shown in Table 2. Then, AGW responds with Location Update Response to PMD (Steps 3 and 4).

3.4. Packet Delivery Operation. In Figure 6, PMD wants to communicate with a particular PMD that is residing in the corresponding gateway (c-AGW). The PMD will send Device ID Request message to AGW (Step 1). Next, the AGW will check whether the GDID belongs to the same domain with the corresponding PMD or not. Note that an AGW can determine this, based on GDID, since GDID contains information about its home domain. Then, AGW sends Location Discovery Request message to c-AGW. The c-AGW will look up its HGR mapping table and reply with Location Discovery Response message to AGW (Steps 2 and 3). Upon the receipt of the Location Discovery Response message from the c-AGW, the AGW will add the information in its mapping table. After that, the AGW responds with Device ID Response message to PMD (Step 4). Now, the data packets will be forwarded to C-PMD via AGW and c-AGW. 


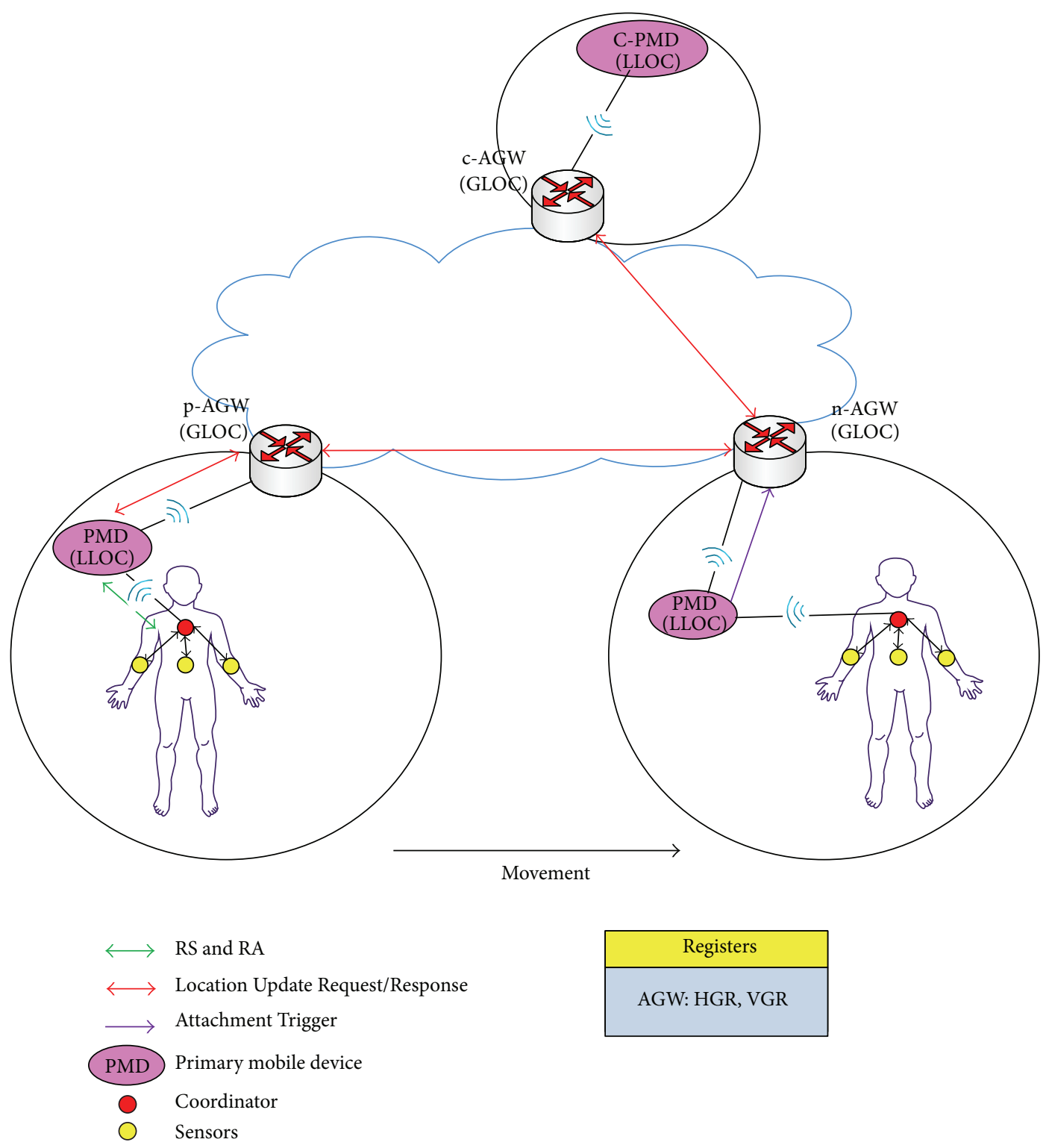

FIGURE 4: Network model for proposed scheme.

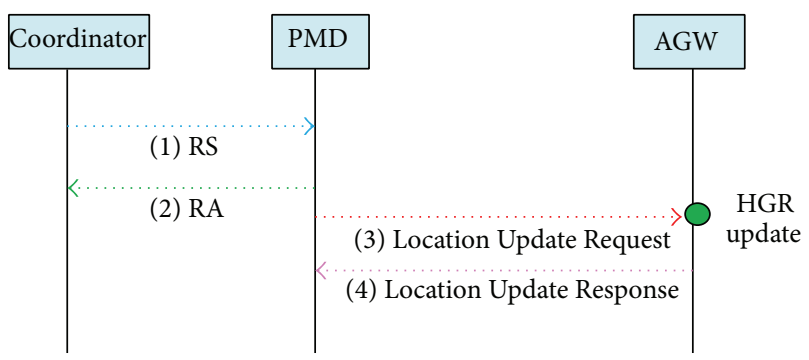

FIGURE 5: Initial registration.

3.5. Handover Operation. When PMD moves from the previous access gateway ( $\mathrm{p}-\mathrm{AGW}$ ) to a new access gateway (n$\mathrm{AGW}$ ) in the same home network domain, the PMD will send
Attachment Trigger to n-AGW (Step 1). After Attachment Trigger, the n-AGW will update its visiting GDID register (VGR) which maintains GDID-LLOC (AID) mapping table as shown in Table 3. Then, $\mathrm{n}-\mathrm{AGW}$ sends Location Update Request message to previous AGW (p-AGW). The p-AGW will update its HGR and responds with Location Update Response message to n-AGW (Steps 2 and 3). The Location Update Response message shall include the information of corresponding-AGW (c-AGW) address, which is recorded in the mapping table of p-AGW.

Now, n-AGW sends a Location Update Request message to $\mathrm{c}-\mathrm{AGW}$ for route optimization. On the reception of the Location Update Request message, c-AGW will update its mapping table and send a Location Update Response to nAGW. n-AGW and c-AGW will now use the optimized route (Steps 4 and 5). 


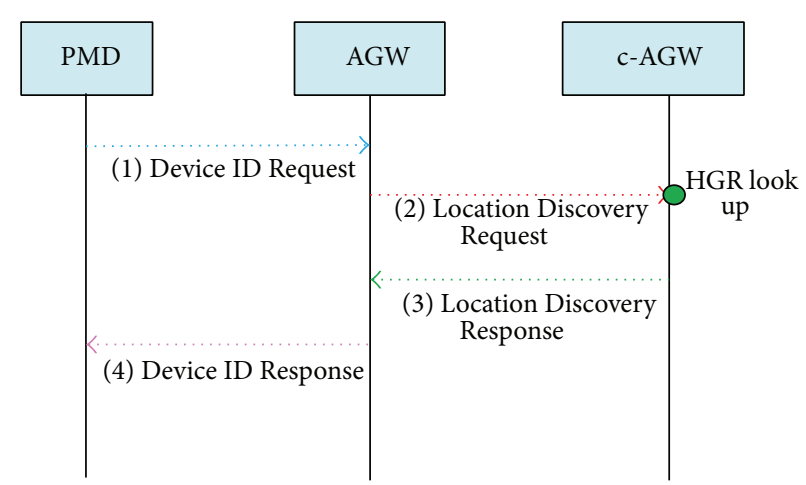

FIgure 6: Packet delivery operation.

TABLE 3: Visiting GDID register (VGR).

\begin{tabular}{cccc}
\hline Number & ID & $\begin{array}{c}\text { LLOC } \\
\text { (in the visited domain) }\end{array}$ & Home domain \\
\hline 1 & GDID1 & LLOC1 (AID) of PMD & GLOC1 \\
& & & (AGW of GDID1) \\
2 & GDID2 & LLOC2 (AID) of PMD & GLOC2 \\
3 & $\ldots$ & $\ldots$ & $\ldots$ \\
\hline
\end{tabular}

\section{Performance Analysis}

In this section, we analyze the performances of candiate mobility management schemes: PMIP-Coordinator, IDLOC-based scheme, and proposed scheme. As performance metrics, we consider the delays associated with registration, packet delivery, and handover delay, since such delays are very impormant in mobility management.

4.1. Analysis Model. We define several notations for analysis and summarize them in the Notations section. We illustrate the considered network model in Figure 8.

In Figure 8, we denote by $T_{x-y}(S)$ the transmission delay of a message with size $S$ from node $x$ to node $y$ via a wireless link. It can be expressed as $T_{x-y}(S)=((1-q) /(1+q))$. $\left(\left(S / B_{w l}\right)+L_{w l}\right)$. In the meantime, we denote by $T_{x-y}\left(S, H_{x-y}\right)$ the transmission delay of a message with size $S$ from node $x$ to node $y$ via a wired link, where $H_{x-y}$ represents the number of wired hops between node $x$ to node $y$. Note that it is expressed as $T_{x-y}\left(S, H_{x-y}\right)=H_{x-y} \cdot\left(\left(S / B_{w}\right)+L_{w}+T_{q}\right)$.

\subsection{Analysis of Registration Delay (RD)}

4.2.1. PMIP-Coordinator. As shown in Figure 1, when Coordinator is attached to MAG, then it sends $R S$ message to MAG by way PMD. After that, the MAG performs AuthenticationAuthorization-Accounting (AAA) query and reply operation with AAA server for authentication for all body sensors. Then, the MAG performs the AAA query operation with AAA server, and then MAG performs aggregated $P B U$ operation with LMA. Then, LMA performs the AAA query and reply operations with AAA server for each body sensor.
After authentication, LMA respond with aggregated $P B A$ to MAG. Now, the MAG responds with aggregated $R A$ message to Coordinator. Accordingly, we get the RD of PMIPCoordinator as follows:

$$
\begin{aligned}
\mathrm{RD}_{\text {PMIP-Coordinator }} \\
=2 T_{\text {C-PMD }}\left(S_{c}\right)+2 T_{\text {PMD-GW }}\left(S_{c}\right) \\
+2 T_{\text {GW-LMA } / \text { DLMA }}\left(S_{c}\right) \\
+N_{S} \times\left\{2 T_{\text {GW-AAA }}\left(S_{c}\right)+2 T_{\text {LMA-AAA }}\left(S_{c}\right)\right\} .
\end{aligned}
$$

4.2.2. ID-LOC-Based Scheme. As shown in Figure 2, when body sensors are attached to PMD, then it performs $R S$ and $R A$ messages to PMD by way of Coordinator. After that, the PMD performs Location Update Request and Response operation with AGW. Then, the AGW also performs the Location Update Request and Response operation with $\mathrm{H}$ DLMA. Accordingly, we get the RD of ID-LOC-based scheme as follows:

$$
\begin{aligned}
\mathrm{RD}_{\mathrm{ID}-\mathrm{LOC}}= & N_{S} \times\left\{2 T_{\mathrm{C}-\mathrm{PMD}}\left(S_{c}\right)\right\} \\
& +2 T_{\mathrm{PMD}-\mathrm{GW}}\left(S_{c}\right)+2 T_{\mathrm{GW}-\mathrm{LMA} / \text { DLMA }}\left(S_{c}\right) .
\end{aligned}
$$

4.2.3. Proposed Scheme. As shown in Figure 5, when Coordinator is attached to $\mathrm{PMD}$, then it performs aggregated $R S$ and $R A$ messages to PMD. After that, the PMD performs Location Update Request and Response operation with AGW. Accordingly, we get the $\mathrm{RD}$ of proposed scheme as follows:

$$
\mathrm{RD}_{\text {Proposed scheme }}=2 T_{\mathrm{C}-\mathrm{PMD}}\left(S_{c}\right)+2 T_{\text {PMD-GW }}\left(S_{c}\right) \text {. }
$$

\subsection{Analysis of Packet Delivery Delay (PDD)}

4.3.1. PMIP-Coordinator. When PMD wants to communicate with particular C-PMD, PMD will forward the data packets to LMA and the LMA will forward the data packet to c-AGW and further to C-PMD. Accordingly, we get the PDD of PMIP-Coordinator as follows:

$$
\begin{aligned}
& \text { PDD }_{\text {PMIP-Coordinator }} \\
& \quad=2 T_{\text {PMD-GW }}\left(S_{d}\right)+2 T_{\text {GW-LMA/DLMA }}\left(S_{d}\right) .
\end{aligned}
$$


4.3.2. ID-LOC-Based Scheme. When PMD wants to communicate with particular C-PMD, PMD will send Device ID Request message to AGW. If there is no information, then AGW performs Location Discovery Request and Response message with C-DLMA. Then, AGW sends Route Setup Request message to c-AGW. After that, the c-AGW performs the GDID Discovery Query and Reply messages with corresponding PMD (C-PMD). After that, the c-AGW responds with Route Setup Complete message to AGW. After that, the AGW responds with Device ID Response message to PMD. Now, the data packets will be forwarded to C-PMD via AGW and c-AGW:

$$
\begin{aligned}
\mathrm{PDD}_{\mathrm{ID}-\mathrm{LOC}}= & 4 T_{\mathrm{PMD}-\mathrm{GW}}\left(S_{c}\right)+2 T_{\mathrm{GW}-\mathrm{LMA} / \mathrm{DLMA}}\left(S_{c}\right) \\
& +2 T_{\mathrm{GW}-\mathrm{GW}}\left(S_{c}\right)+2 T_{\mathrm{PMD}-\mathrm{GW}}\left(S_{d}\right) \\
& +T_{\mathrm{GW}-\mathrm{GW}}\left(S_{d}\right) .
\end{aligned}
$$

4.3.3. Proposed Scheme. When PMD wants to communicate with particular C-PMD, PMD will send Device ID Request message to AGW. If there is no information, then AGW performs Location Discovery Request and Response message with c-AGW. After that, the AGW responds with Device ID Response message to PMD. Now, the data packets will be forward to C-PMD via AGW and c-AGW:

$$
\begin{aligned}
\mathrm{PDD}_{\text {Proposed Scheme }}= & 2 T_{\text {PMD-GW }}\left(S_{c}\right)+2 T_{\mathrm{GW}-\mathrm{GW}}\left(S_{c}\right) \\
& +2 T_{\text {PMD-GW }}\left(S_{d}\right)+T_{\mathrm{GW}-\mathrm{GW}}\left(S_{d}\right) .
\end{aligned}
$$

\subsection{Analysis of Handover Delay (HD)}

4.4.1. PMIP-Coordinator. As shown in Figure 1, when the Coordinator is detached from p-MAG, then p-MAG will send aggregated DeReg message to LMA. When Coordinator is attached to $\mathrm{n}-\mathrm{MAG}$, then it sends $R S$ message to $\mathrm{n}$ MAG by way PMD. After that, the n-MAG performs $A A A$ query and reply operation with AAA server for authentication for all body sensors. Then, the n-MAG performs the $A A A$ query operation with AAA server, and then nMAG performs aggregated $P B U$ operation with LMA. Then, LMA performs the AAA query and reply operations with AAA server for each body sensor. After authentication, LMA responds with aggregated $P B A$ to $n-M A G$. The handover tunnel is established between n-MAG and LMA. After tunnel establishment, the n-MAG responds with aggregated $R A$ message to Coordinator. Accordingly, we get the HD of PMIPCoordinator as follows:

$$
\begin{aligned}
\mathrm{HD}_{\text {PMIP-Coordinator }} & \\
= & 2 T_{\text {C-PMD }}\left(S_{c}\right)+2 T_{\text {PMD-GW }}\left(S_{c}\right) \\
& +4 T_{\text {GW-LMA/DLMA }}\left(S_{c}\right) \\
& +N_{S} \times\left\{2 T_{\text {GW-AAA }}\left(S_{c}\right)+2 T_{\text {LMA-AAA }}\left(S_{c}\right)\right\} \\
& +T_{\text {GW-LMA/DLMA }}\left(S_{d}\right) .
\end{aligned}
$$

4.4.2. ID-LOC-Based Scheme. As shown in Figure 2, when PMD is attached with n-AGW, then PMD will send Attachment Trigger to n-AGW. After that, the n-AGW performs Device Context Request and Reply messages with pAGW. Then, p-AGW performs Location Update Request and Response messages with H-DLMA. Accordingly, we get the HD of ID-LOC-based scheme as follows:

$$
\begin{aligned}
\mathrm{HD}_{\mathrm{ID}-\mathrm{LOC}}= & T_{\mathrm{PMD}-\mathrm{GW}}\left(S_{c}\right)+2 T_{\mathrm{GW}-\mathrm{GW}}\left(S_{c}\right) \\
& +2 T_{\mathrm{GW}-\text { LMA } / \text { DLMA }}\left(S_{c}\right)+T_{\mathrm{GW}-\mathrm{GW}}\left(S_{d}\right) .
\end{aligned}
$$

4.4.3. Proposed Scheme. As shown in Figure 7, when PMD is attached with n-AGW, then PMD will send Attachment Trigger to n-AGW. After that, the n-AGW performs Location Update Request and Response messages with p-AGW. Accordingly, we get the HD of proposed scheme as follows:

$$
\begin{aligned}
& \mathrm{HD}_{\text {Proposed Scheme }} \\
& \quad=T_{\text {PMD-GW }}\left(S_{c}\right)+2 T_{\mathrm{GW}-\mathrm{GW}}\left(S_{c}\right)+T_{\mathrm{GW}-\mathrm{GW}}\left(S_{d}\right) .
\end{aligned}
$$

\section{Numerical Results and Discussion}

Based on the analytical equations given in Section 4, we compare the performances of the considered mobility management schemes. In the numerical results, the default value of each parameter has been configured as follows, referring to [11]; that is, $H_{\text {DLMS-DLMS }}=5, H_{\mathrm{GW} \text {-LMA/DLMS }}=5, H_{\mathrm{GW}-\mathrm{GW}}=$ $5, H_{\mathrm{GW}-\mathrm{AAA}}=5$, and $H_{\mathrm{LMA}-\mathrm{AAA}}=5$, and $L_{w l}=10(\mathrm{~ms})$, $L_{w}=2(\mathrm{~ms}), q=0.5, N_{S}=10, T_{q}=5(\mathrm{~ms}), S_{c}=96$ (bytes), $S_{d}=200$ (bytes), $B_{w l}=11$ (Mbps), and $B_{w}=100$ (Mbps), where $N_{S}$ denotes the number of sensors in the network. Among the various parameters, we note that $L_{w l}, T_{q}, N_{S}$, and $H_{\mathrm{GW} \text {-LMA/DLMA }}$ can depend on the network conditions. Thus, we evaluate the performances of the considered schemes by varying the values of these parameters.

5.1. Registration Delay. We show the impact of the delay of wireless links $\left(L_{w l}\right)$ on the registration delay in Figure 9. We can see that the registration delay increases linearly as $L_{w l}$ becomes larger in every considered scheme. In particular, the PMIP-Coordinator mobility scheme is more sensitive to the delay of wireless links than the ID-LOC mobility scheme, since they exchange the signaling messages for the registration over wireless links and also perform AAA query operation with AAA server for each body sensor. The PMIPCoordinator also performs $P B U / P B A$ operation with LMA for binding. While ID-LOC mobility scheme performs better than PMIP-Coordinator, this is because there is no AAA query operation with AAA server, since it performs $R S / R A$ messages with PMD for each body sensors over wireless link. We observe that the proposed scheme performs best among the candidate schemes. This is because the Coordinator performs $R S / R A$ messages with $\mathrm{PMD}$ and also there is no binding operation with DLMA, since the binding operation performs with AGW.

Figure 10 compares the registration delays of candidate schemes by varying the average queuing delay $\left(T_{q}\right)$ at each 


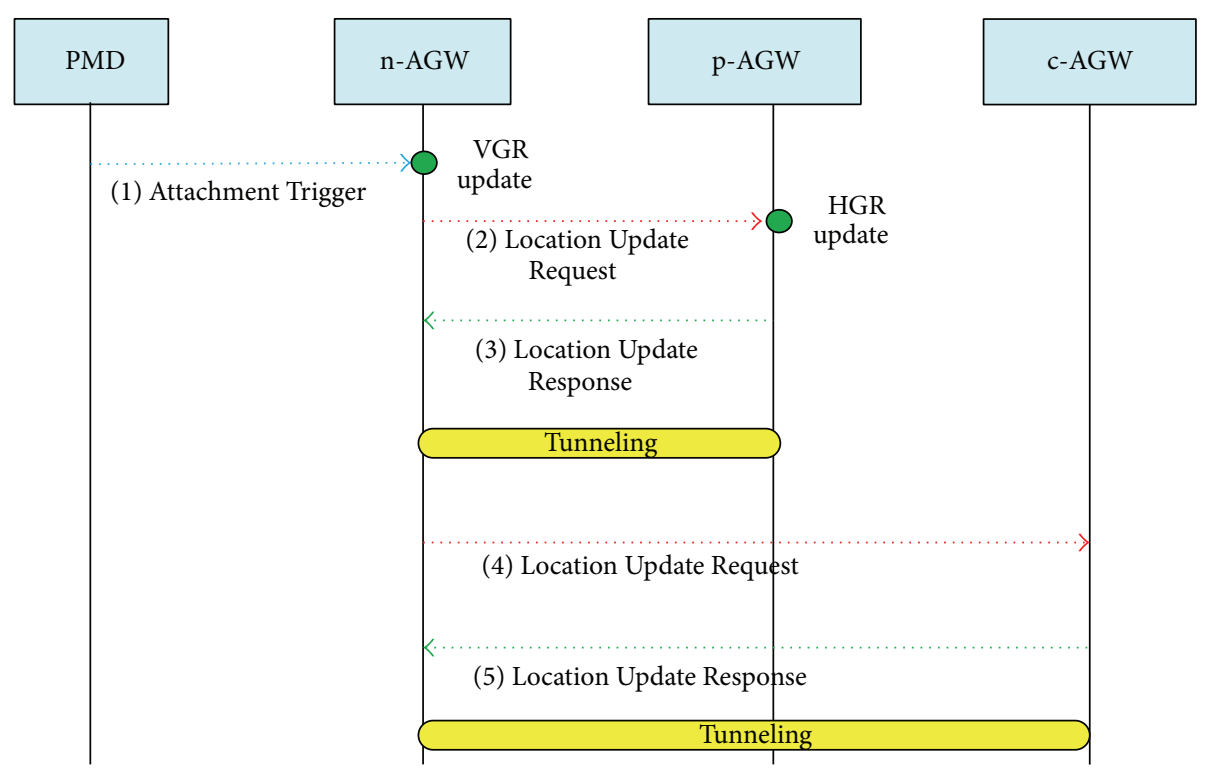

FIGURE 7: Handover operation.

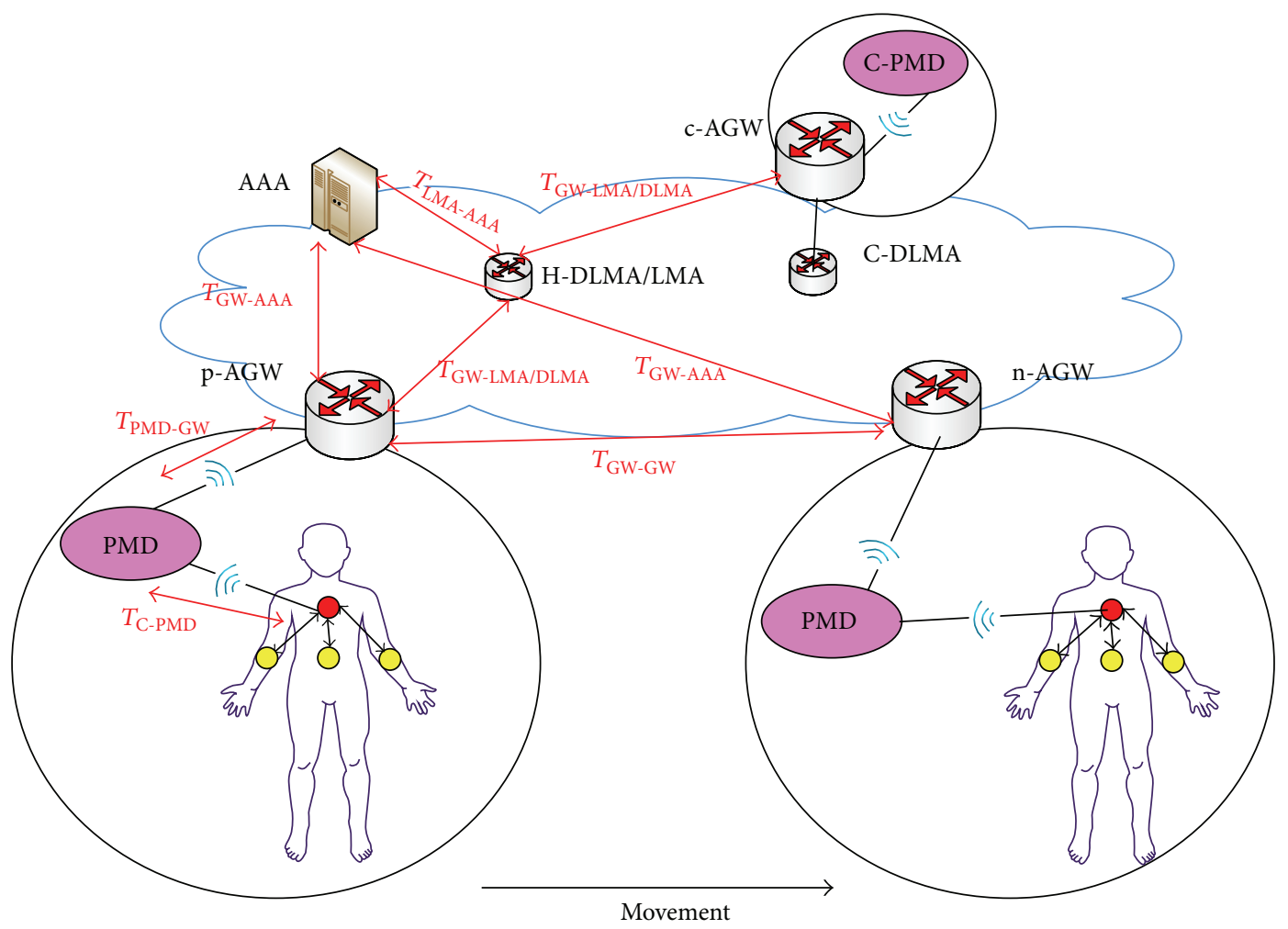

FIGURE 8: Network model for performance analysis.

node. For the two schemes, PMIP-Coordinator and ID-LOCbased scheme, the registration delay increases linearly as $T_{q}$ increase because the two schemes exchange the signaling messages with AAA, LMA, and DLMA over the wired network. In contrast, proposed scheme is not affected by the average queuing delay at all since it exchanges the signaling messages for the registration over wireless links only. We can see that the proposed scheme performs well compared to the existing schemes.

We next illustrate the registration delay for different number of sensors in the network $\left(N_{S}\right)$ in Figure 11. We observe that the PMIP-Coordinator gives worse performances than the ID-LOC-based scheme. This is because of the signaling messages for authentication with AAA server by GW and 


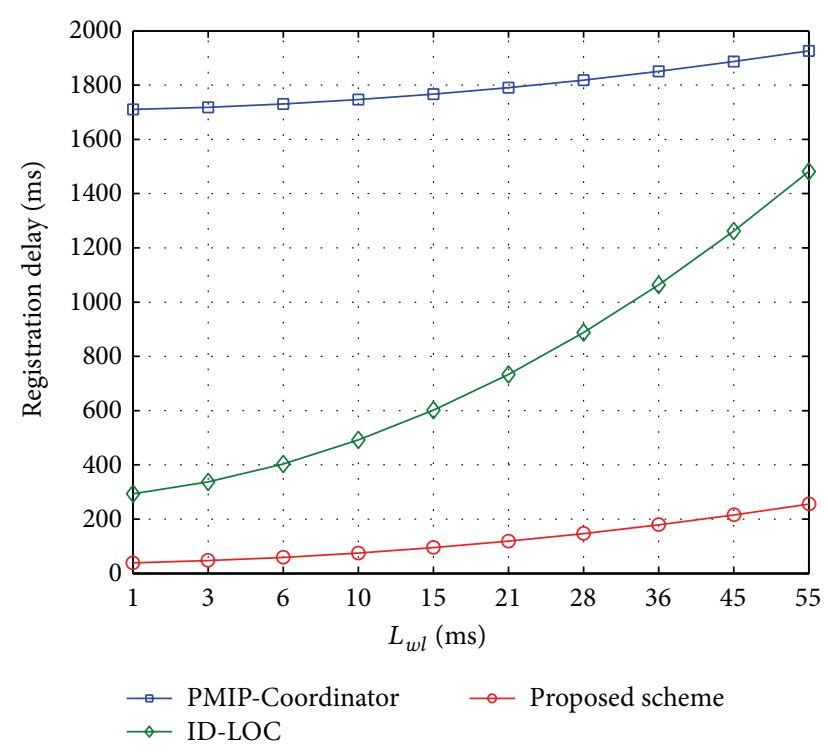

FIGURE 9: Impact of $L_{w l}$ on the registration delay.

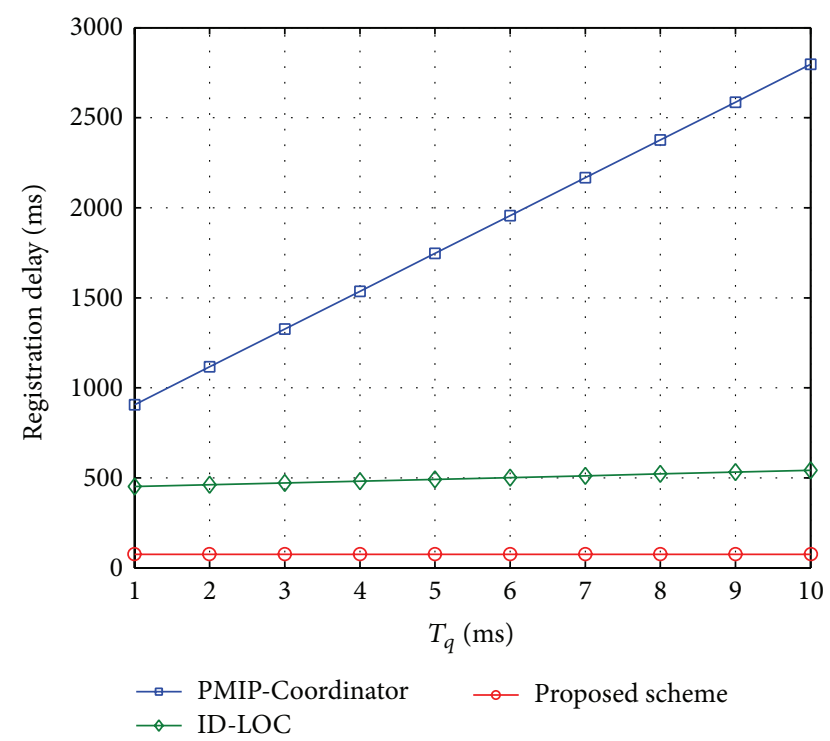

FIgURE 10: Impact of $T_{q}$ on the registration delay.

LMA for each body sensor. In contrast, the proposed scheme is not affected by the number of sensors. This is because only Coordinator can exchange the RS/RA messages with PMD on behalf of body sensors.

5.2. Packet Delivery Delay. We illustrate the packet delivery delay for different hop counts between GW and LMA/DLMA $\left(H_{\mathrm{GW}-\mathrm{LMA} / \mathrm{DLMA}}\right)$ in Figure 12 . We can see that $H_{\mathrm{GW}-\mathrm{LMA} / \mathrm{DLMA}}$ affects the performances of the existing mobility schemes significantly. We observe that ID-LOC-based scheme performs poorly compared to the PMIP-Coordinator scheme. This is because there is query operation before data delivery, while PMIP-Coordinator relies on LMA in the distance for the registration and the data delivery. We can see in the figure

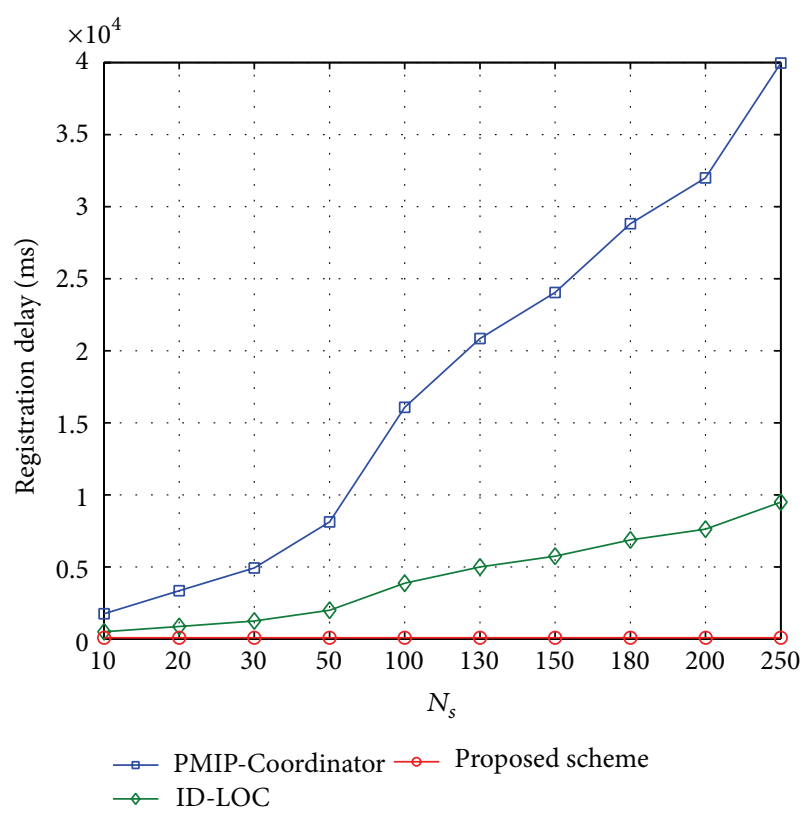

FIgURE 11: Impact of $N_{S}$ on the registration delay.

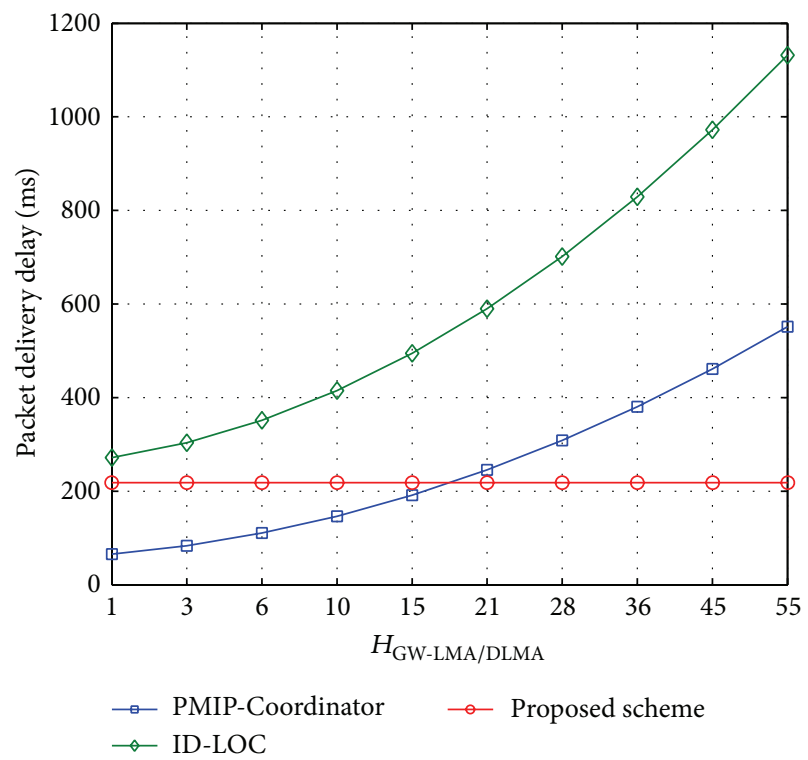

FIGURE 12: Impact of $H_{\text {GW-LMA/DLMA }}$ on the packet delivery delay.

that the proposed scheme is not affected with $H_{\mathrm{GW} \text {-LMA/DLMA }}$, since AGW maintains HGR and VGR.

5.3. Handover Delay. The delay of wireless links $\left(L_{w l}\right)$ gives a significant impact on the handover delay for the three candidate schemes, as shown in Figure 13. In particular, the PMIPCoordinator mobility scheme is more sensitive to the delay of wireless links than the ID-LOC mobility scheme, since the signaling messages exchanged over the wireless links spends much time in configuring a new care-of-address through the duplicate address detection procedure by handover. The PMIP-Coordinator also performs $P B U / P B A$ operation with 
TABLE 4: Improvement of proposed scheme from existing schemes in average percentage.

\begin{tabular}{lcccccc}
\hline & \multicolumn{2}{c}{ Registration delay } & \multicolumn{2}{c}{ Handover delay } & \multicolumn{2}{c}{ Packet delivery delay } \\
Parameters & $\begin{array}{c}\text { From } \\
\text { PMIP-Coordinator }\end{array}$ & From ID-LOC & $\begin{array}{c}\text { From } \\
\text { PMIP-Coordinator }\end{array}$ & From ID-LOC & $\begin{array}{c}\text { From } \\
\text { PMIP-Coordinator }\end{array}$ & From ID-LOC \\
\hline$L_{w l}$ & $6.7 \%$ & $15.6 \%$ & $8.5 \%$ & $67.2 \%$ & $\mathrm{X}$ & $\mathrm{X}$ \\
$T_{q}$ & $4.5 \%$ & $15.13 \%$ & $8.2 \%$ & $66 \%$ & $\mathrm{X}$ & $\mathrm{X}$ \\
$N_{s}$ & $1 \%$ & $4.1 \%$ & $\mathrm{X}$ & $\mathrm{X}$ & $\mathrm{X}$ \\
$H_{\text {GW-LMA/DLMA }}$ & $\mathrm{X}$ & $\mathrm{X}$ & $6.4 \%$ & $42.6 \%$ & $136.1 \%$ & $44.7 \%$ \\
\hline
\end{tabular}

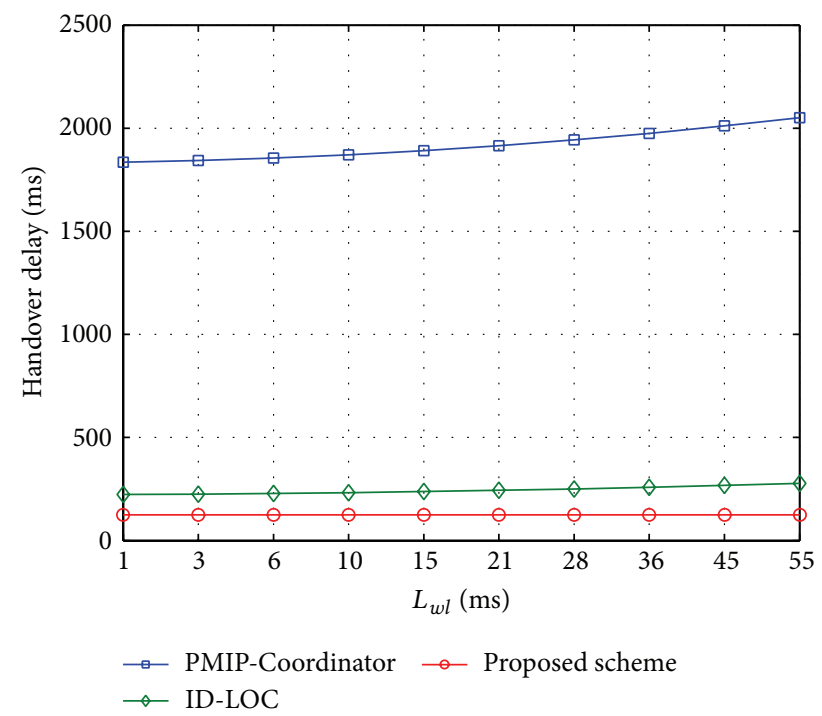

FIGURE 13: Impact of $L_{w l}$ on the handover delay.

LMA for binding. While ID-LOC mobility scheme performs better than PMIP-Coordinator, this is because there is no AAA query operation with AAA server, since it performs $R S / R A$ messages with PMD for each body sensor over wireless link. We can see in the figure that proposed scheme performs best among the candidate schemes.

Figure 14 shows the impact of the average queuing delay $\left(T_{q}\right)$ on the handover delay. The delay rises up linearly as $T_{q}$ increases in all the schemes. We observe that the PMIPCoordinator performs worst while consuming much time in the duplicate address detection procedure.

We illustrate the handover delay for different hop counts between GW and LMA/DLMA ( $\left.H_{\text {GW-LMA/DLMA }}\right)$ in Figure 15. We can see that $H_{\text {GW-LMA/DLMA }}$ affects the performances of the existing mobility schemes significantly, since they rely on LMA/DLMA in the distance for the registration and the data delivery.

Table 4 summarizes the improvement of the proposed scheme from the existing schemes in terms of average percentage based on parameters.

\section{Conclusion}

In this paper, we proposed a group-based mobility management scheme, which is based on ID/LOC separation concept for ID-based communications with location-based

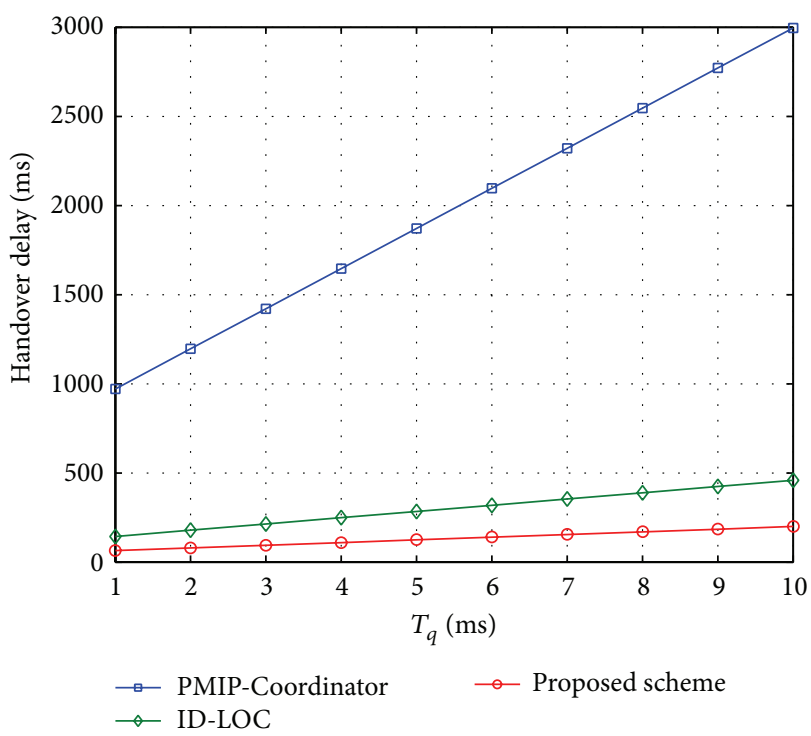

FIGURE 14: Impact of $T_{q}$ on the handover delay.

routing to reduce the number of control messages. In the proposed scheme, the sensor node and PMD have globally unique device identifiers (GDIDs). Each GDID contains the information of the associated home network domain. For handover support, each access gateway maintains its home GDID register and its visiting GDID register. In addition, only the Coordinator can exchange $R S / R A$ messages with PMD instead of each sensor. The numerical analysis shows that the proposed scheme can reduce the registration delay, packet delivery delay, and the handover delay significantly, compared to the existing mobility schemes.

\section{Notations for Numerical Analysis}

$S_{c}: \quad$ Size of control packets (bytes)

$S_{d}: \quad$ Size of data packets (bytes)

$B_{w l}:$ Wireless bandwidth (Mbps)

$L_{w l}$ : Wireless link delay (ms)

$B_{w}$ : Wired link bandwidth (Mbps)

$L_{w}$ : Wired link delay (ms)

$H_{x-y}$ : Hop count between nodes $x$ and $y$

$T_{q}: \quad$ Average queuing delay at each node (ms)

$q$ : $\quad$ Wireless link failure probability

$N_{S}$ : Number of sensors in the network. 


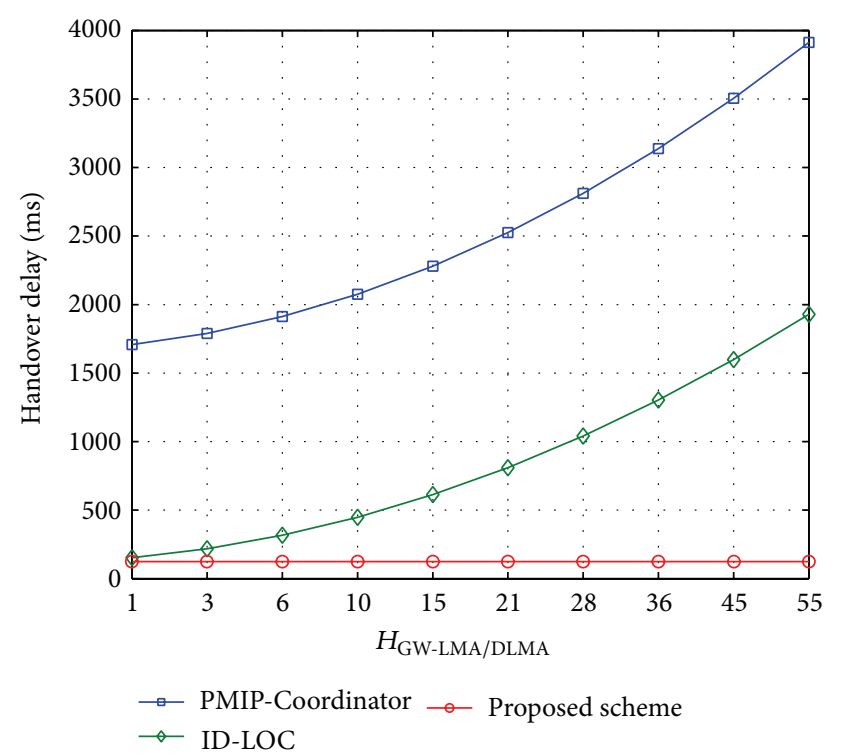

FIgURE 15: Impact of $H_{\text {GW-LMA/DLMA }}$ on the handover delay.

\section{Conflict of Interests}

The authors declare that there is no conflict of interests regarding the publication of this paper.

\section{References}

[1] J. Xing and Y. Zhu, "A survey on body area network," in Proceedings of the 5th International Conference on Wireless Communications, Networking and Mobile Computing (WiCOM '09), September 2009.

[2] R. Cavallari, F. Martelli, R. Rosini, C. Buratti, and R. Verdone, "A survey on wireless body area networks: technologies and design challenges," IEEE Communications Surveys \& Tutorials, vol. 16, no. 3, pp. 1635-1657, 2014.

[3] M. M. Alam and E. B. Hamida, "Surveying wearable human assistive technology for life and safety critical applications: standards, challenges and opportunities," Sensors, vol. 14, no. 5, pp. 9153-9209, 2014.

[4] N. Kushalnagar, G. Montenegro, and C. Schumacher, "IPv6 over low-power wireless personal area networks (6LoWPANs): overview, assumptions, problem statement, and goals," IETF RFC 4919, 2007.

[5] G. Montenegro, N. Kushalnagar, J. Hui, and D. Culler, "Transmission of IPv6 packets over IEEE 802.15.4 networks," IETF RFC4944, 2007.

[6] D. Johnson, C. Perkins, and J. Arkko, "Mobility support in IPv6," IETF RFC 3775, 2004.

[7] S. Gundavelli, K. Leung, V. Devarapalli, K. Chowdhury, and B. Patil, "Proxy mobile IPv6," IETF RFC 5213, 2008.

[8] V. Devarapalli, R. Wakikawa, A. Petrescu, and P. Thubert, "Network mobility (NEMO) basic support protocol," IETF RFC 3963, 2005.

[9] Y.-S. Chen, C.-S. Hsu, and H.-K. Lee, "An enhanced group mobility protocol for 6lowpan-based wireless body area networks," IEEE Sensors Journal, vol. 14, no. 3, pp. 797-807, 2014.
[10] J. Kim, J. Lee, H. Kyu Kang, D. Sun Lim, C. Seon Hong, and S. Lee, "An ID/locator separation-based mobility management architecture for WSNs," IEEE Transactions on Mobile Computing, vol. 13, no. 10, pp. 2240-2254, 2014.

[11] C. Makaya and S. Pierre, "An analytical framework for performance evaluation of IPv6-based mobility management protocols," IEEE Transactions on Wireless Communications, vol. 7, no. 3, pp. 972-983, 2008. 

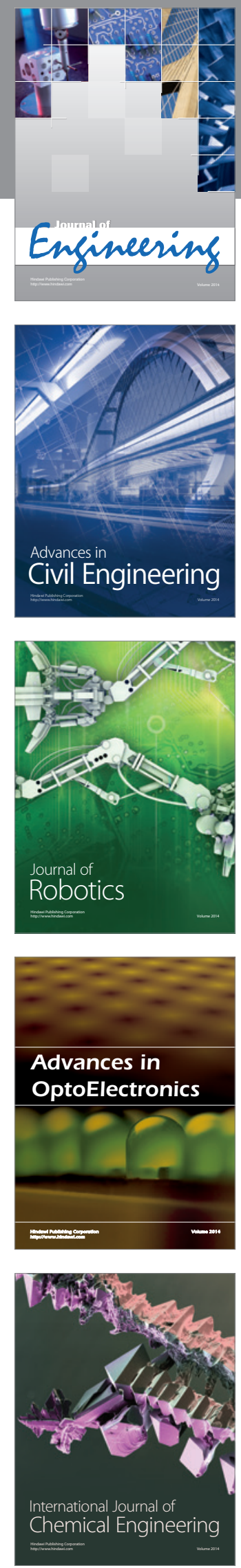

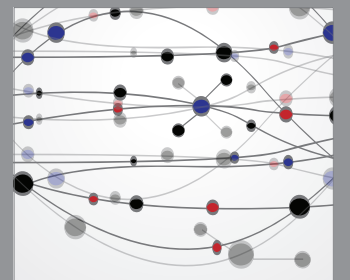

The Scientific World Journal
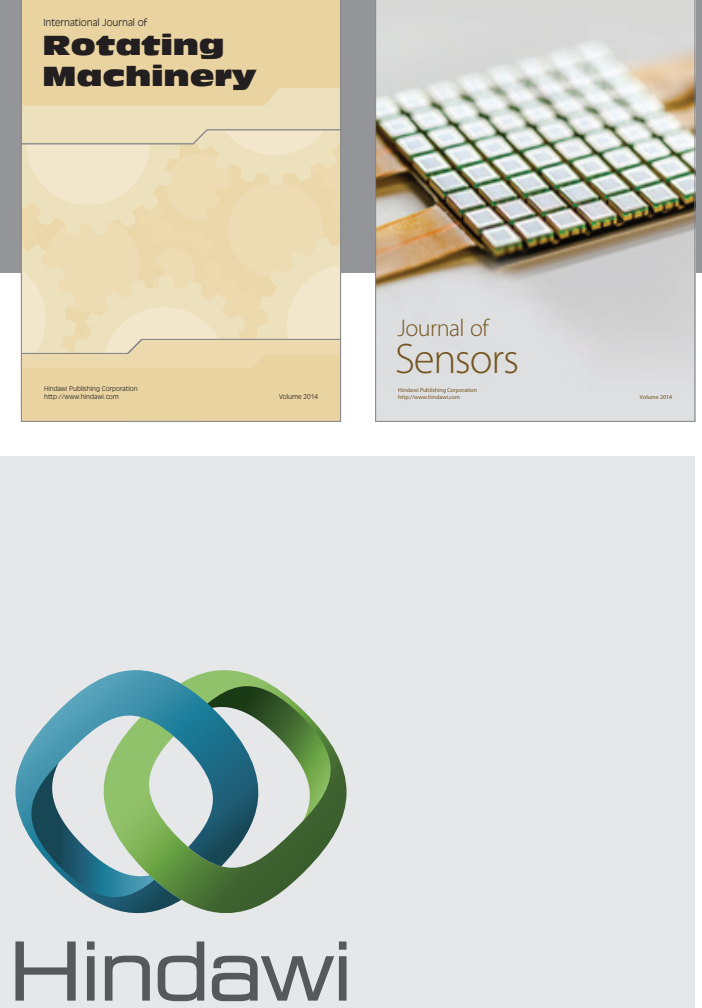

Submit your manuscripts at http://www.hindawi.com
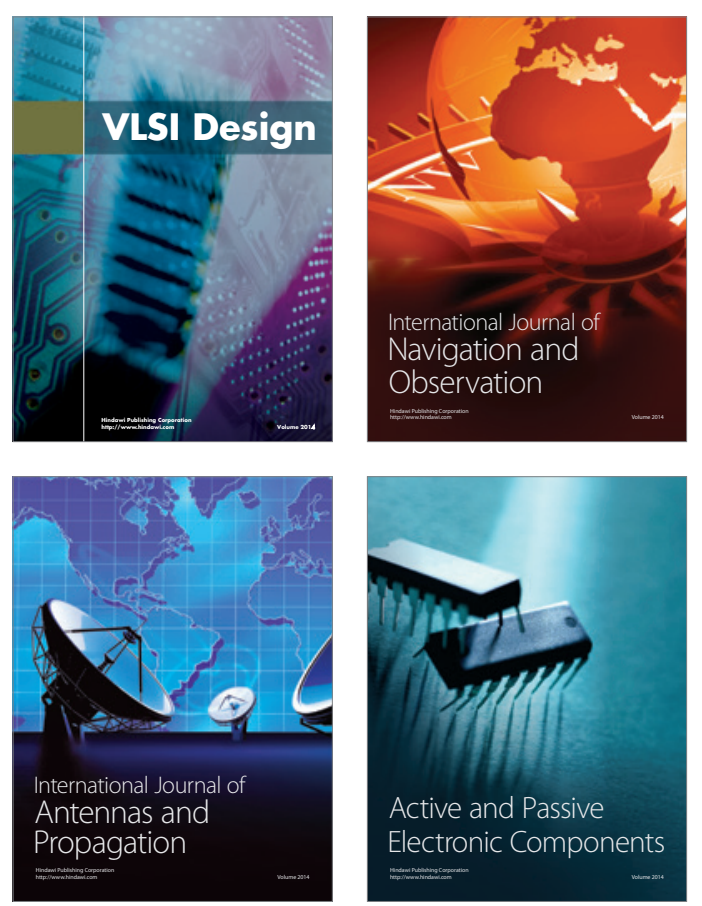
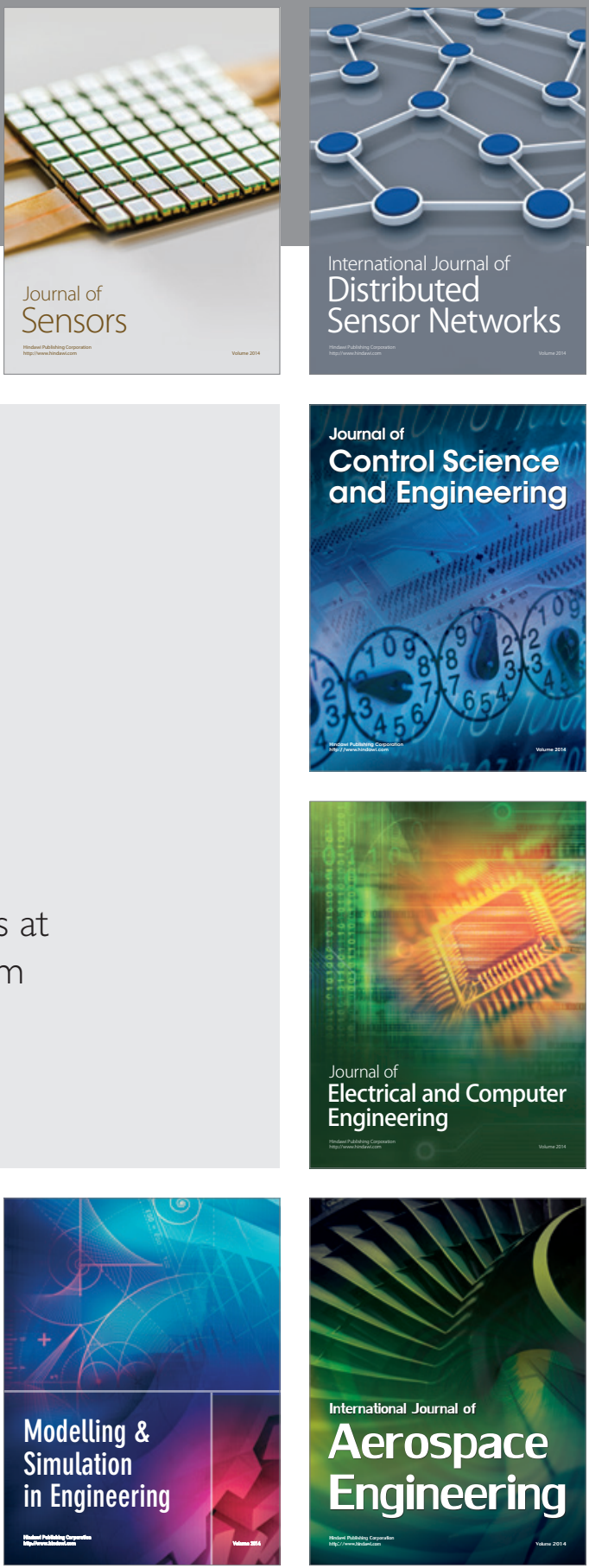

Journal of

Control Science

and Engineering
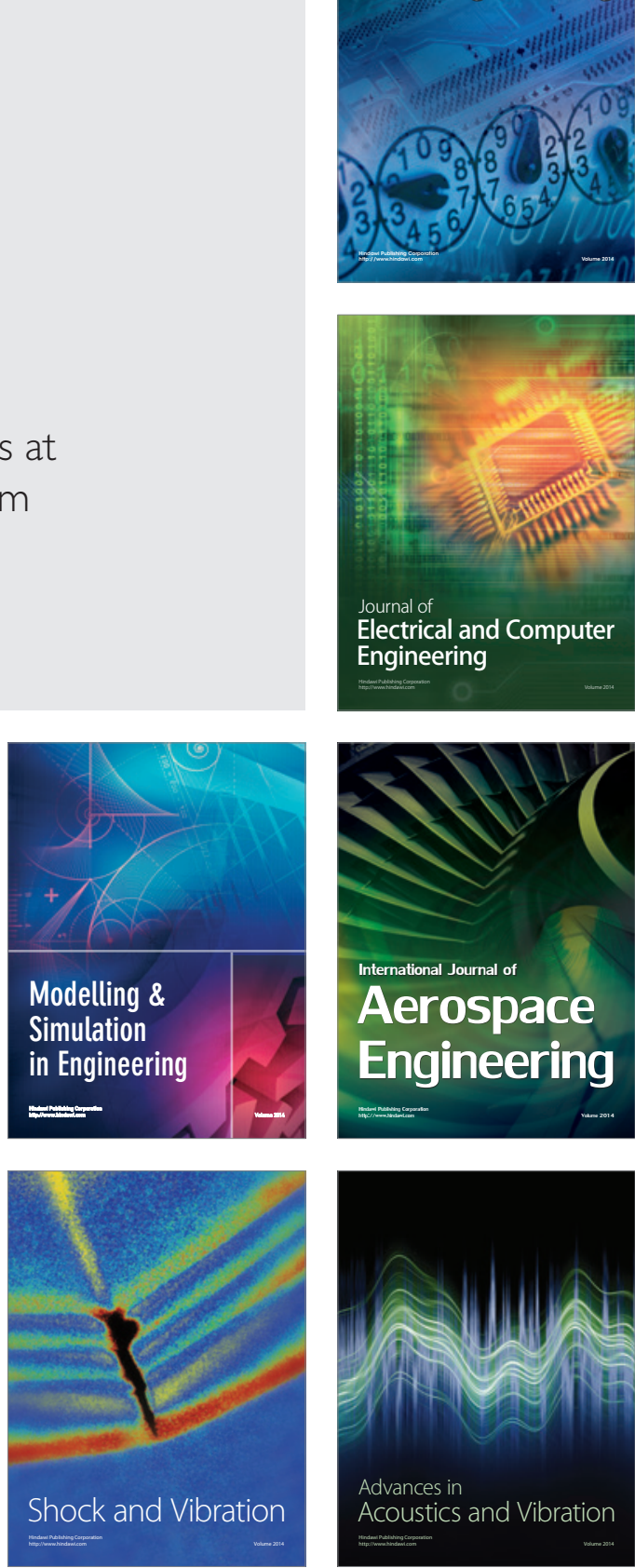